\title{
Origins of the long-range exciton diffusion in perovskite nanocrystal films: photon recycling vs exciton hopping
}

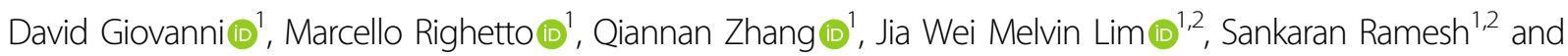 \\ Tze Chien Sum (1)
}

\begin{abstract}
The outstanding optoelectronic performance of lead halide perovskites lies in their exceptional carrier diffusion properties. As the perovskite material dimensionality is reduced to exploit the quantum confinement effects, the disruption to the perovskite lattice, often with insulating organic ligands, raises new questions on the charge diffusion properties. Herein, we report direct imaging of $>1 \mu \mathrm{m}$ exciton diffusion lengths in $\mathrm{CH}_{3} \mathrm{NH}_{3} \mathrm{PbBr}_{3}$ perovskite nanocrystal (PNC) films. Surprisingly, the resulting exciton mobilities in these PNC films can reach $10 \pm 2 \mathrm{~cm}^{2} V^{-1} \mathrm{~s}^{-1}$, which is counterintuitively several times higher than the carrier mobility in 3D perovskite films. We show that this ultralong exciton diffusion originates from both efficient inter-NC exciton hopping (via Förster energy transfer) and the photon recycling process with a smaller yet significant contribution. Importantly, our study not only sheds new light on the highly debated origins of the excellent exciton diffusion in PNC films but also highlights the potential of PNCs for optoelectronic applications.
\end{abstract}

\section{Introduction}

The remarkable long and balanced carrier diffusion properties of lead halide perovskites (LHPs) underpin their unprecedented optoelectronic device performance. In 3D (bulk) LHP systems, balanced carrier diffusion lengths exceeding $1 \mu \mathrm{m}$ have been reported ${ }^{1,2}$, with signatures of ballistic transport in early dynamics ${ }^{3,4}$. Similarly, robust transport properties have recently been demonstrated in quasi-2D LHP systems, enabled by longrange exciton diffusion ${ }^{5}$ and ultrafast spin-preserving exciton transport ${ }^{6}$ over a range of hundreds of $\mathrm{nm}$. Therefore, understanding and optimizing the robust transport properties of LHPs hold the key to their successful application in devices.

Correspondence: Tze Chien Sum (Tzechien@ntu.edu.sg)

1 Division of Physics and Applied Physics, School of Physical and Mathematical Sciences, Nanyang Technological University (NTU), 21 Nanyang Link,

Singapore 637371, Singapore

2Energy Research Institute @NTU (ERI@N), Interdisciplinary Graduate School,

Nanyang Technological University, 50 Nanyang Avenue, S2-B3a-01,

Singapore 639798, Singapore

These authors contributed equally: David Giovanni, Marcello Righetto
Recently, low-dimensional LHPs [e.g., nanocrystals (NCs), quantum dots (QDs), nanorods, and nanowires] have attracted much interest with the promise of further boosting the LHP-based device performance. For instance, the power conversion efficiencies of photovoltaic devices could be improved by carefully tailoring multiple exciton generation $(\mathrm{MEG})^{7,8}$ and hot-carrier cooling processes ${ }^{9,10}$. Moreover, the external quantum efficiencies of LHP-based light-emitting devices have been greatly enhanced by leveraging quantum confinement, resulting in increased absorption cross-sections and photoluminescence quantum yields (PLQYs). However, preserving the excellent transport properties is the toughest hurdle to overcome with the concomitant dimensionality reduction. Firstly, the nature of the carrier transport is altered, as quantum confinement effects enhance excitonic properties, thereby bringing additional factors into play such as the electronic coupling strength between neighboring nanostructures. Secondly, additional sources of static and dynamic disorder are inadvertently introduced due to the size distribution,

\section{(c) The Author(s) 2021}

(c) (i) Open Access This article is licensed under a Creative Commons Attribution 4.0 International License, which permits use, sharing, adaptation, distribution and reproduction cc) in any medium or format, as long as you give appropriate credit to the original author(s) and the source, provide a link to the Creative Commons license, and indicate if changes were made. The images or other third party material in this article are included in the article's Creative Commons license, unless indicated otherwise in a credit line to the material. If material is not included in the article's Creative Commons license and your intended use is not permitted by statutory regulation or exceeds the permitted use, you will need to obtain permission directly from the copyright holder. To view a copy of this license, visit http://creativecommons.org/licenses/by/4.0/. 
ligand interdigitation, site vacancies, and generally disordered energy landscape.

Interparticle excitonic interactions in II-VI semiconductor QD films and superlattices have been studied since the first report by Kagan et al. ${ }^{11,12}$. Despite extensive studies and tailoring of the microscopic mechanism of energy transfer (encompassing downhill Förster mediated transfer in an inhomogeneous ensemble), less attention is paid to the exciton spatial propagation properties in QD films. In 2014, Tisdale et al. ${ }^{13}$ reported elegant direct measurement of diffusion lengths in II-VI core-shell QD films based on PL imaging. Unfortunately, small diffusion lengths $(\sim 20-30 \mathrm{~nm})$ were reported for $\mathrm{CdSe} / \mathrm{CdZnS}$ QDs, where the disordered energy landscapes induce subdiffusive exciton transport. Hitherto, these factors have severely constrained the realization of hyper-structured confined-yet-electronically coupled films, which could significantly advance QDbased PVs, display/LED technologies, and lasing fields.

Amid such impasses, lead halide perovskite NCs (PNCs) recently emerged as promising candidates to achieve longrange transport in quantum-confined nanostructures owing to their intrinsically defect-tolerant electronic structure $^{14-16}$ and weakly size-dependent properties ${ }^{17}$. Here, we report the direct measurement of exceptional long-range energy transport of $>1 \mu \mathrm{m}$ in methylammonium lead bromide $\left(\mathrm{MAPbBr}_{3}\right) \mathrm{PNC}$ films measured with a direct PL imaging method, surpassing previous reports on other nanostructures. We demonstrate tuning of the exciton mobility in our PNC films up to $D=10 \pm 2 \mathrm{~cm}^{2}$ $\mathrm{V}^{-1} \mathrm{~s}^{-1}$ by simple modification of the organic ligands. Using a phenomenological model, we elucidate the quantitative contributions of inter-NC exciton hopping $(\mathrm{EH})$ and photon recycling (PR) processes to the ultralong exciton diffusion length. Our results not only demonstrate the unprecedented micron-scale diffusion length of excitons in PNCs but also deepen the understanding of the fundamental mechanisms underpinning long-range energy transport in PNC films.

\section{Results}

\section{Imaging of exciton diffusion in PNC films}

$\mathrm{MAPbBr}_{3} \mathrm{PNCs}$ were synthesized with different organic ligands (Fig. 1a) by slightly modifying a previously reported ligand-assisted reprecipitation method (LARP) ${ }^{18}$. Briefly, PNCs were synthesized by direct precipitation of perovskite precursors in a mixed ligand/benzyl alcohol/ toluene phase. The length of the amine ligands was modified while the precursor to ligand molar ratio was kept constant to ensure a consistent synthetic procedure and achieve similar sizes of the PNCs. In this study, we used oleic acid and three different amine ligands: hexylamine, octylamine, and oleylamine (hereafter abbreviated as hexyl, octyl, and oleyl, respectively). Figure $1 \mathrm{~b}$ presents the absorption and photoluminescence (PL) spectra of the various purified colloidal PNCs. Excitonic absorption resonances peak at approximately $2.40 \mathrm{eV}(517 \mathrm{~nm})$ and can be more clearly identified by the minima in the $2^{\text {nd }}$ derivative of the absorption spectra [Supplementary Information, Fig. S1]. Minor differences between samples occur due to the difference in the solubility of the ligands in the antisolvent phase, yielding slightly different growth kinetics ${ }^{19}$. The samples exhibit a strong PL peak at approximately $2.36 \mathrm{eV}(526 \mathrm{~nm})$ with minimal Stokes shift. The size distribution from the TEM micrograph (Fig. S2) confirms PNCs with average diameters distributed between 5.7 and $7.7 \mathrm{~nm}$, as shown in Table 1. Notably, the narrow FWHM of $\sim 91 \mathrm{meV}(\sim 20 \mathrm{~nm})$, despite the broad size distribution of the PNCs, is in agreement with the well-known weak size dependency ${ }^{17}$. Given the reported exciton Bohr radius of $\mathrm{MAPbBr}_{3}$ of $\sim 2 \mathrm{~nm}^{20,21}$ and the bulk emission energy $\hbar \omega_{\text {bulk }}$ of $\sim 2.30 \mathrm{eV}(\sim 540 \mathrm{~nm})^{22}$, our PNCs can be considered weakly confined excitonic systems.

Based on their defect tolerance ${ }^{14-16}$ and weak size dependency ${ }^{17}$, we envisage the possibility of long-range exciton diffusion in PNC films. Comparatively, forerunner studies on II-VI QD films determined that exciton diffusion/transport in such quantum confined systems is largely inhibited by traps, either on the single dot scale, where the surface exhibits midgap energy levels acting as carrier traps, or on the ensemble scale, where the size dependence of energy levels yields larger dots (i.e., lower energy sites) within the ensemble that act as exciton traps $^{13}$. On the other hand, the defect-tolerant properties and size-insensitive electronic structures of PNCs can be leveraged; these properties largely limit the impact of electronic disorder on exciton transport. Moreover, the high PLQY and small Stokes shift in PNCs also promote efficient inter-NC energy transfer (ET) processes, both radiative ET (i.e., PR) and nonradiative ET [e.g., Förster resonance energy transfer (FRET)]. Thanks to these properties, PNCs stem as ideal candidates to achieve long diffusion lengths in quantum-confined systems.

To test our hypothesis, we spatially measured the exciton transport inside PNC films by using a steady-state modification of the PL profile expansion method introduced by Tisdale et al. ${ }^{13}$-Fig. 1c. A continuous-wave $(\mathrm{CW})$ diode laser with a photon energy of $2.62 \mathrm{eV}(473 \mathrm{~nm})$ was focused to an $\sim 2 \mu \mathrm{m}$ spot on the PNC films. The exciton population dynamics $n(x, y, t)$ in the steady-state condition can be described by the following differential equation:

$$
\frac{\partial n(x, y, t)}{\partial t}=0=G(x, y)-\frac{n(x, y)}{\tau}+D \nabla^{2} n(x, y)
$$

The first term on the right-hand side represents the generation rate, which is proportional to the pump intensity profile [i.e., $G(x, y) \propto I_{\text {pump }}(x, y)$, which is a 


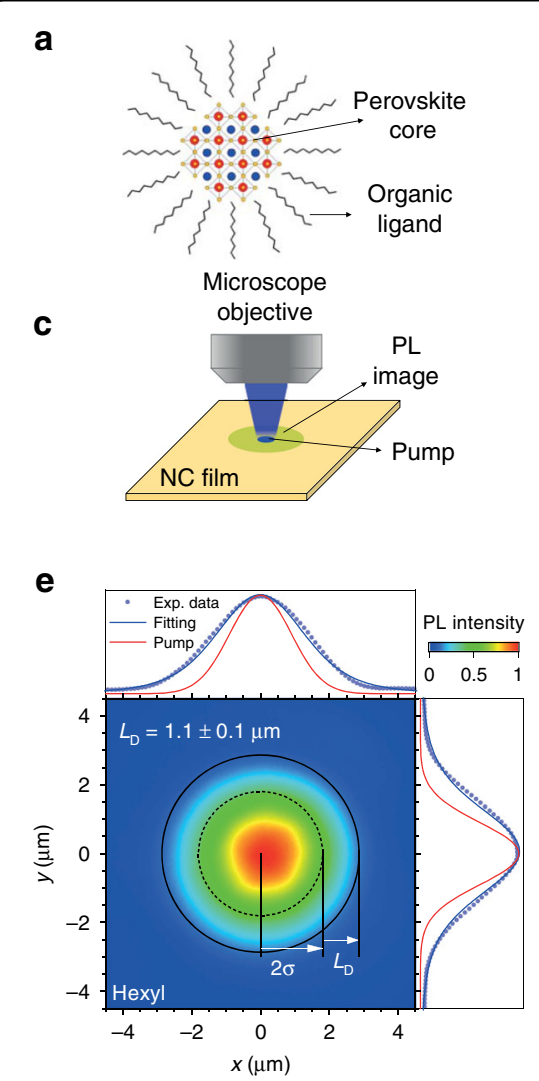

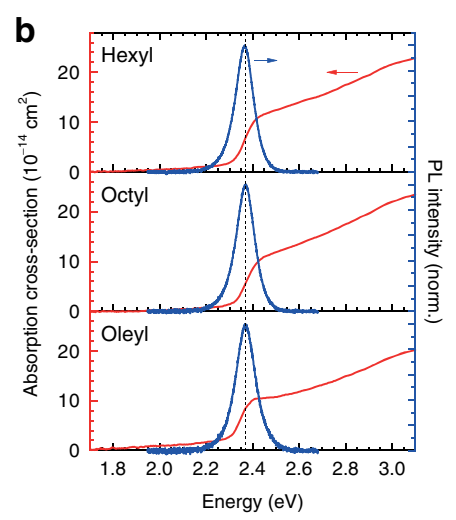

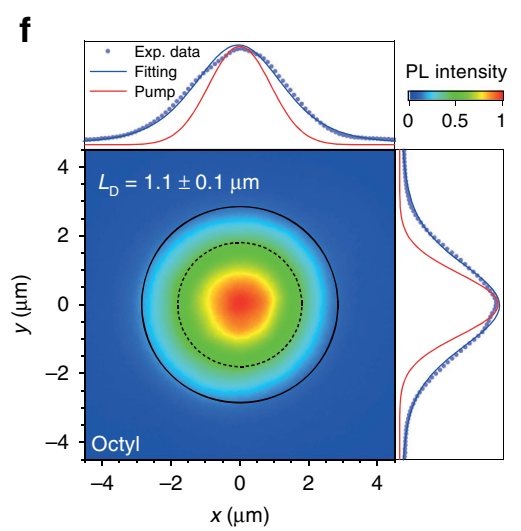

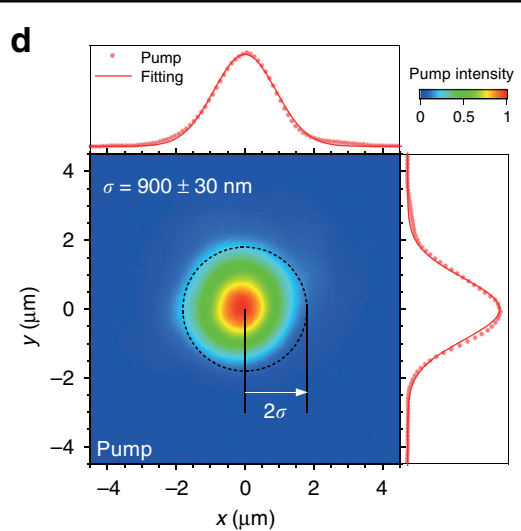

g

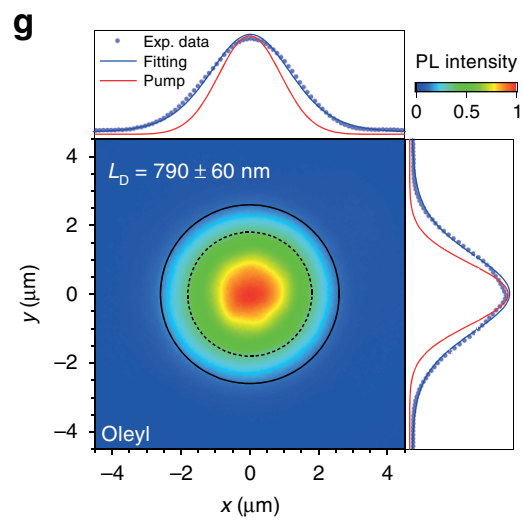

Fig. 1 Linear absorption and PL in PNC samples. (a) Illustration of our PNC samples. (b) Absorption and PL spectra of colloidal MAPbBr ${ }_{3}$ PNCs with

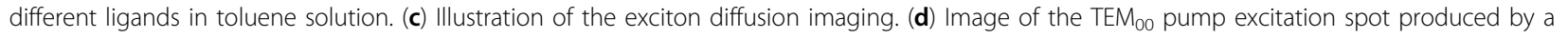
$473 \mathrm{~nm}$ CW laser, with $\boldsymbol{\sigma}=900 \pm 30 \mathrm{~nm}$ (FWHM =2.1 $\pm 0.1 \mu \mathrm{m}$ ). The pump profile was fitted with a 2D Gaussian function. (e-g) PL images of the PNC films with different ligands, whose profiles were fitted with Eq. (2). The $x$ - and $y$-cross-sections of the PL (blue) and pump (red) are shown for comparison. The dotted line in (b) corresponds to the maximum PL wavelength. The dotted and solid lines in the ( $\mathrm{d}-\mathrm{g}$ ) contour color plots correspond to circles with radii of $2 \boldsymbol{\sigma}$ and $2 \boldsymbol{\sigma}+\boldsymbol{L}_{\mathrm{D}}$, respectively

Gaussian $\mathrm{TEM}_{00}$ mode]; the second term describes the exciton recombination with lifetime $\tau$; and lastly, the third term describes the exciton diffusion in two dimensions, with diffusion coefficient $D$. The solution for the differential equation is given by:

$$
I_{\mathrm{PL}}(x, y) \propto \int_{-\infty}^{\infty} \mathrm{d} x^{\prime} \int_{-\infty}^{\infty} \mathrm{d} y^{\prime} \exp \left(-\frac{x^{\prime 2}+y^{\prime 2}}{2 \sigma^{2}}\right) K_{0}\left(\frac{\sqrt{\left[x^{\prime}-x\right]^{2}+\left[y^{\prime}-y\right]^{2}}}{L_{\mathrm{D}}}\right)
$$

Details of the derivation can be found in Supplementary Note 1 . Here, $I_{\mathrm{PL}}$ is the 2D PL image profile observed in our experiment; $K_{0}(x)$ is the zeroth-order modified Bessel function of the second kind; and $L_{\mathrm{D}}=\sqrt{D \tau}$ is the exciton diffusion length.

Equation (2) satisfactorily reproduces the 2D PL image profiles of our PNC films, as confirmed by the plots of their $x$ - and $y$ - cross-sections (Fig. 1e-g).
Table 1 Properties of $\mathrm{MAPbBr}_{3}$ PNCs and their films

\begin{tabular}{llll}
\hline & Hexyl & Octyl & Oleyl \\
\hline Size $(\mathrm{nm})$ & $6 \pm 2$ & $6 \pm 2$ & $8 \pm 3$ \\
PLQY (\%) & $55 \pm 2$ & $65 \pm 2$ & $40 \pm 2$ \\
Exciton energy (eV) & 2.40 & 2.42 & 2.39 \\
Absorption cross-section at & $23 \pm 2$ & $23 \pm 3$ & $20 \pm 2$ \\
$400 \mathrm{~nm}\left(10^{-14} \mathrm{~cm}^{2}\right)^{*}$ & & & \\
Effective lifetime $(\mathrm{ns})^{* *}$ & $58 \pm 5$ & $42 \pm 3$ & $41 \pm 4$ \\
Diffusion length $(\mu \mathrm{m})$ & $1.1 \pm 0.1$ & $1.1 \pm 0.1$ & $0.79 \pm 0.06$ \\
Diffusion coefficient $\left(\mathrm{cm}^{2} \mathrm{~s}^{-1}\right)$ & $0.20 \pm 0.03$ & $0.27 \pm 0.04$ & $0.15 \pm 0.02$ \\
Exciton mobility $\left(\mathrm{cm}^{2} \mathrm{~V}^{-1} \mathrm{~s}^{-1}\right)$ & $8 \pm 1$ & $10 \pm 2$ & $5.9 \pm 0.8$ \\
\hline
\end{tabular}

The size distributions, PLQYs, exciton resonance energies, and other measured parameters of the PNCs are given. Diffusion lengths, diffusion coefficients, and exciton mobilities are given for the corresponding films

${ }_{* *}^{*}$ Measured by Poisson distribution fitting (Supplementary Note 2)

${ }^{* *}$ Measured at a low pump fluence, corresponding to a population of $\mathrm{N} \sim 0.07$ per $\mathrm{NC}$ 
The fitting results are reported in Table 1 . From the fitting, unprecedented ultralong diffusion lengths $\left(L_{\mathrm{D}}\right)$ exceeding $1 \mu \mathrm{m}$ are obtained, which is unusual for such quantum confined systems. For comparison, typical inorganic QD systems (e.g., CdSe QDs) exhibit exciton diffusion lengths of only a few tens of $\mathrm{nm}^{13,23}$. The larger values in PNCs correspond to a diffusion coefficient up to $0.27 \pm 0.04 \mathrm{~cm}^{2} \mathrm{~s}^{-1}$, which is equivalent to an exciton mobility of $\mu=e D / k_{\mathrm{B}} T=10 \pm$ $2 \mathrm{~cm}^{2} \mathrm{~V}^{-1} \mathrm{~s}^{-1}$. Such neutral exciton diffusion is different from charge carrier diffusion in bulk semiconductors. Nevertheless, it is still interesting to note that this exciton mobility is comparable to/larger than the reported typical carrier mobility for bulk 3D perovskite films ${ }^{24}$, i.e., averages of $2.4 \pm 1.1 \mathrm{~cm}^{2} \mathrm{~V}^{-1} \mathrm{~s}^{-1}$ and $\sim 8.6 \mathrm{~cm}^{2} \mathrm{~V}^{-1} \mathrm{~s}^{-1}$ for $\mathrm{MAPbI}_{3}$ and $\mathrm{MAPbBr}_{3}$ thin films, respectively. These results imply that the reduction of the transport properties due to quantum confinement and insulating ligands is fully compensated by the efficient inter-QD ET in PNC films.

These excellent exciton transport properties in PNC films could stem from two possible mechanisms. The first is the radiative (or trivial) ET mechanism, i.e., PR, where emitted photons from one $\mathrm{NC}$ are reabsorbed by the neighboring NCs. PR has also been reported to enhance not only the transport properties in other types of perovskite systems ${ }^{25-31}$ but also LED performances ${ }^{32}$.
The second mechanism is the EH via nonradiative ET between neighboring NCs [e.g., FRET, Dexter energy transfer (DET), or other mechanisms]. Herein, we seek to explicate the quantitative contributions of these two mechanisms in our PNC films.

\section{Contribution from PR}

Figure 2a, b illustrates the PR mechanism and signatures. For a single NC emitter (no PR), the absorption of a photon creates an exciton, which will recombine radiatively after an average time $\langle\tau\rangle=\tau_{0}$ (Fig. 2a). This photon could then be immediately captured by the time-resolved detection system for lifetime measurement. However, in the case of NC ensembles (with PR), the photon will be reabsorbed and reemitted by the neighboring NCs an average $\langle M\rangle$ number of times before leaving the ensemble and being recorded by the time-resolved detection equipment (Fig. 2b). In this case, the detector will measure an increased lifetime of $\langle\tau\rangle=\tau_{0}(1+\langle M\rangle)$.

Another signature of PR is spectral redshift due to the higher absorption of blue light for any system with PLQY below unity during the reabsorption and reemission processes. To elucidate this process in our PNC system, we performed a time-resolved PL study on the colloidal PNC systems. Colloidal PNCs provide an ideal platform to understand the PR process for several reasons. First, the concentration of NCs per unit volume in the colloidal

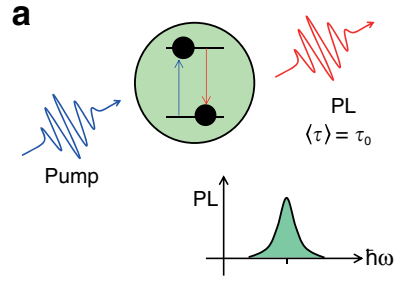

Single particle (no photon recycling)

b

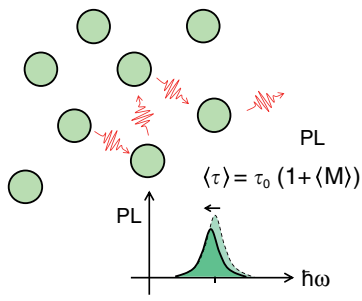

Ensemble (w/ photon recycling)

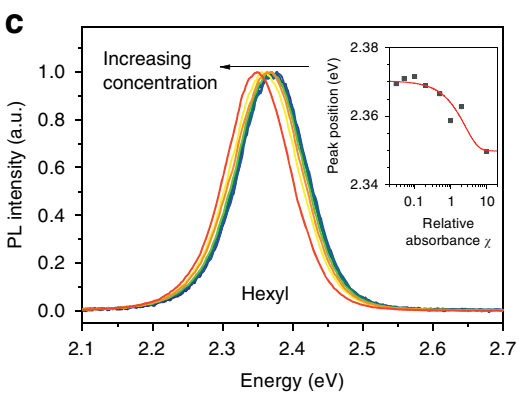

d

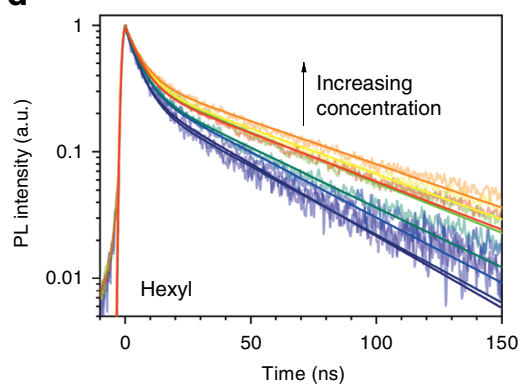

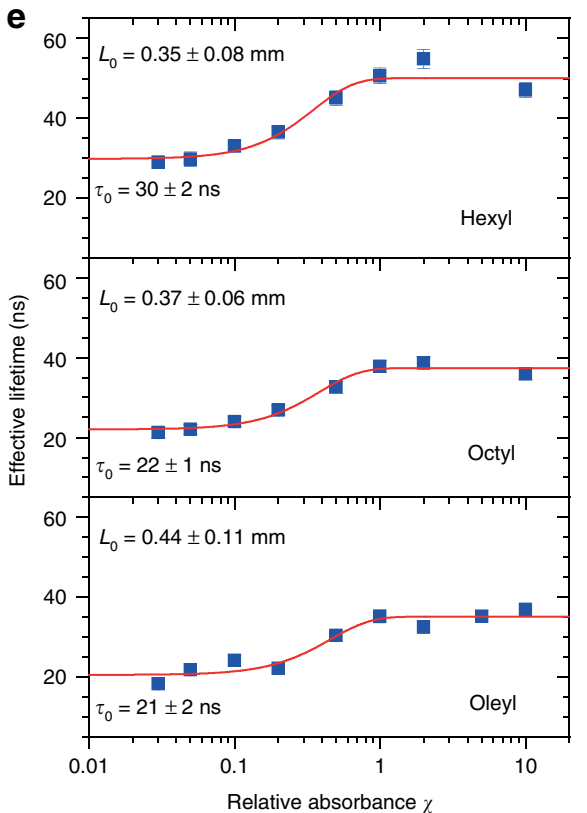

Fig. 2 Effect of PR in colloidal PNCs. (a, b) Illustration of the effect of PR on the PL spectra and lifetimes. (c) Redshifting PL spectra and (d) increasing PL lifetime of our colloidal PNCs. The inset in fig. (c) shows the peak position as a function of concentration, as fitted by the reabsorption model (Supplementary Note 3). (e) Effective PL lifetimes of our PNCs with different ligands as a function of the relative colloidal absorbance (X). The data were fitted with our PR model. The samples were excited with a $3.1 \mathrm{eV}$ pump with a fluence corresponding to $\mathbf{N} \sim 0.6$ excitons per NC 
system ensures sufficient distance between NCs to prevent nonradiative ET processes from occurring. The typical NC concentration obtained from our in situ solution-processed synthesis is $\sim 0.6 \mu \mathrm{M}$, corresponding to an average interparticle distance of $\sim 0.14 \mu \mathrm{m}$. For comparison, nonradiative ET processes such as FRET or DET could only occur within a distance of at most a few $\mathrm{nm}^{33-35}$.

Second, the colloidal system also provides a facile platform for modulating the NC concentration, and hence controlling the PR mean free path (MFP) and the average number of reabsorption-reemission processes $M$. These variables are crucial for quantifying the contribution of PR in PNC films. Indeed, PR signatures were revealed as we modulated the concentration of our colloidal NCs. As the concentration increases, the photon MFP inside the solution decreases, resulting in an increased $M$. Namely, a photon that originates from a given depth inside a cuvette will be recycled (i.e., absorbed and reemitted) a number of times before it can emerge from the solution to be detected. Figure 2c, d shows clear spectral redshifts of the PL central wavelength, together with increasing PL lifetime with increasing colloidal concentration.

To provide a quantitative description of this process, we modeled the PR in a colloidal system photoexcited by a pump traveling along the positive $z$-direction. The colloidal solution is assumed to be situated inside a cuvette with its two interfaces located at $z=0$ and $z=$ $L_{\text {cuv }}$ (i.e., $L_{\text {cuv }}$ is the cuvette thickness, $1 \mathrm{~mm}$ ). A segment of the random movement of a photon in 3-dimensional space inside the colloidal solution can be described by $L^{2}=\left\langle x^{2}\right\rangle+\left\langle y^{2}\right\rangle+\left\langle z^{2}\right\rangle$, where $L$ is the photon MFP inside the colloidal solution, while $\left\langle x^{2}\right\rangle,\left\langle y^{2}\right\rangle$, and $\left\langle z^{2}\right\rangle$ are the square averages of the displacement of the photon in the $x-, y-$ and $z$-directions, respectively. Given that the photon is emitted in a random direction, the square average of the displacement will be the same for all directions, i.e., $\left\langle x^{2}\right\rangle=\left\langle y^{2}\right\rangle=\left\langle z^{2}\right\rangle$. Hence, the root-meansquare displacement in the $z$-direction for every PR process is given by:

$$
z_{\mathrm{RMS}}=\sqrt{\left\langle z^{2}\right\rangle}=\frac{L}{\sqrt{3}}
$$

Based on random walk theory, a photon originating from a depth $z$ inside the cuvette will experience PR on average $M$ times before escaping, i.e., $M(z)=\left(z / z_{\mathrm{RMS}}\right)^{2}=3 z^{2} / L^{2}$. The initial exciton distribution $n(z)$ created by the photoexcitation is described by $n(z)=n_{0} \exp \left(-\sigma_{\text {pump }} c z\right)$, where $\sigma_{\text {pump }}$ and $c$ are the absorption cross-section at the pump energy and the concentration of the PNCs, respectively. Taking the average of $M$ across the initial photon population, we obtain:

$$
M=\frac{3}{L^{2}} \frac{\int_{0}^{L_{\text {cuv }}} z^{2} \exp \left(-\sigma_{\text {pump }} c z\right) \mathrm{d} z}{\int_{0}^{L_{\text {cuv }}} \exp \left(-\sigma_{\text {pump }} c z\right) \mathrm{d} z}=6 \frac{\sigma_{\mathrm{PL}}^{2}}{\sigma_{\text {pump }}^{2}}\left(1-\frac{A \exp (-A)(1+A / 2)}{1-\exp (-A)}\right)
$$

where $A=\sigma_{\text {pump }} c L_{\text {cuv }}$ is the absorption of the system; $\sigma_{\mathrm{PL}}$ is the sample absorption cross-section at the PL energy; and the definition $L=\left(\sigma_{\mathrm{PL}} c\right)^{-1}$ has been used. Therefore, the apparent lifetime of the system $\langle\tau\rangle$ due to PR, as a function of the relative concentration of the colloidal solution, is given by:

$$
\langle\tau\rangle=\tau_{0}(1+\langle M\rangle)=\tau_{0}\left[1+6 \xi^{2}\left(1-\frac{A_{0 X} \exp \left(-A_{0} X\right)\left(1+A_{0 X} / 2\right)}{1-\exp \left(-A_{0} X\right)}\right)\right]
$$

Here, we define $A_{0}$ as the standard absorbance at a given arbitrary standard concentration $c_{0} ; \tau_{0}$ is the intrinsic lifetime without PR; $\xi \equiv \sigma_{P L} / \sigma_{\text {pump }}$ is the ratio of the absorption cross-sections at the PL and pump energies; and $X \equiv A / A_{0}=c / c_{0}$ is the relative absorbance (or equivalent relative concentration) of the colloidal solution with respect to the defined standard. The details of the derivation are provided in Supplementary Note 4. Equation (5) was then used to fit the measured PL effective lifetimes (Supplementary Note 5) of our colloidal NCs as a function of $X$, with $A_{0}, \mathcal{\xi}$, and $\tau_{0}$ as the three fitting parameters. The results are presented in Fig. 2e, where our model successfully describes the observed lifetime trends. From the fitting, we estimated the photon MFP at the standard concentration $\left(\lambda_{0}\right)$ to be in the range of $400-500 \mu \mathrm{m}$. Since $\lambda$ is inversely proportional to the colloidal concentration, these values of $\lambda_{0}$ could be used as a standard parameter to quantify the effect of PR in our systems, as long as their relative concentrations are known (i.e., $\lambda=\lambda_{0} / \chi$ ).

A further validation of this model stems from the consistency of our results, verified by other independent experiments. For instance, Fig. 1b shows the linear absorption cross-section ratio to be $\sim 8 \times 10^{-14} \mathrm{~cm}^{2}$ and $\sim 2 \times 10^{-13} \mathrm{~cm}^{2}$ around the PL region and at $400 \mathrm{~nm}$, respectively. This agrees with the fitting value of $\xi \sim 0.34$ obtained for all our samples. Additionally, we estimated the relative concentration ratio of the NCs in films and in solution $\left(X_{\text {film }}\right)$ to be $\sim 10^{2}$ to $10^{3}$ (ratio of the linear absorption coefficient around $520 \mathrm{~nm}$ ). Using these values together with the fitting results, our model accurately estimates all the PNC film lifetimes at the given $\chi_{\text {film }}$ for all 3 types of ligands, which are in agreement with the timeresolved PL experimental values. The details are provided in Supplementary Note 6 . It is also noteworthy that this model is not only applicable to our PNC systems but also could be applied generally to other systems (e.g., colloidal CdSe QDs and Rh6G organic dye solution, Fig. S3). 

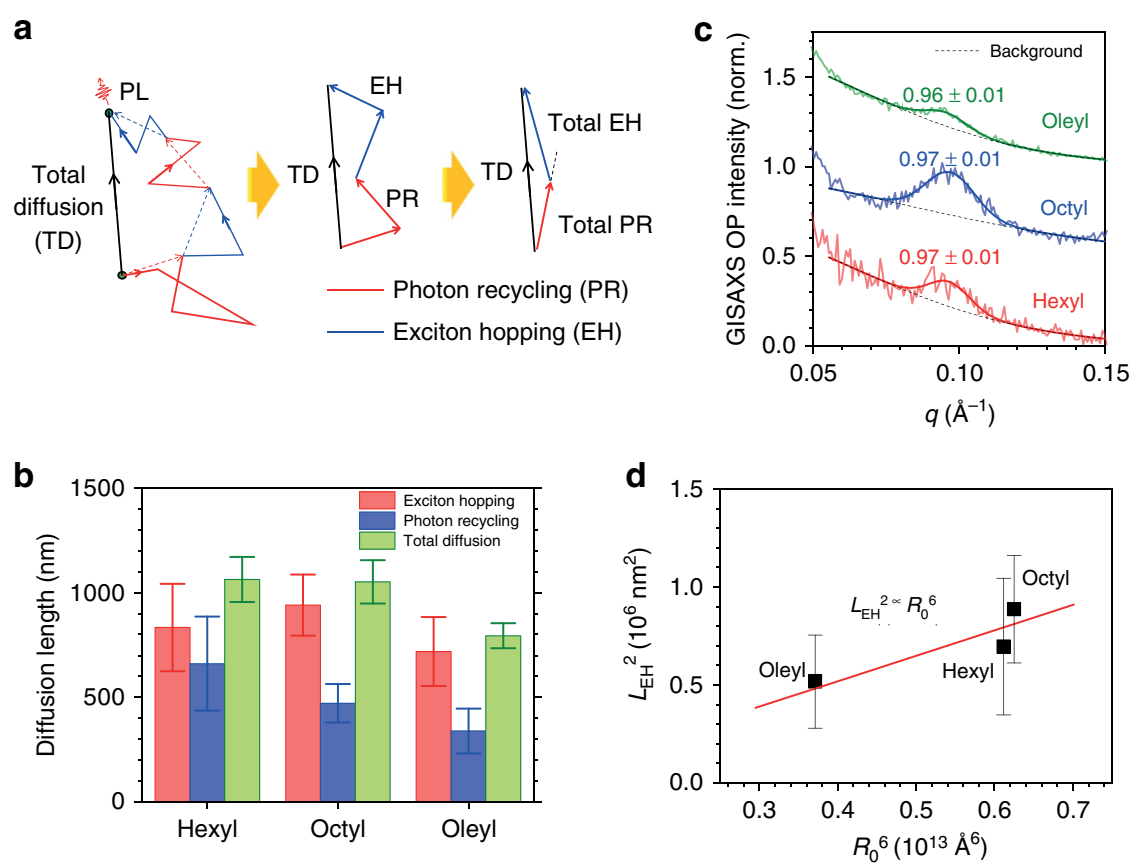

Fig. 3 Distinguishing EH and PR in PNC films. (a) Illustration of the EH and PR contributions to the total diffusion length (TD), which could be represented as vector addition. (b) Estimated quantitative contributions of EH and PR in our PNC films with different ligands. (c) Grazing incidence small-angle X-ray scattering (GISAXS) out-of-plane (OP) measurement for our PNC films. The positions of the peaks are labeled. (d) Correlation between the EH range $\left(\boldsymbol{L}_{\mathrm{EH}}\right)$ and FRET range $\left(\boldsymbol{R}_{0}\right)$, fitted with the Smoluchowski-Einstein relation (red line)

\section{Distinguishing the EH and PR contributions in PNC films}

We proceed to quantify the individual contributions from $\mathrm{PR}$ and $\mathrm{EH}$ to the observed ultralong diffusion length in our PNC films. In this case, the relative contributions of the two possible diffusion mechanisms $(\mathrm{EH}$ and PR) can be described in the framework of vectorial addition - Fig. 3a. We consider a scenario of exciton diffusion consisting of a series of $\mathrm{PR}$ and $\mathrm{EH}$ processes with respective total displacement vectors of $\overrightarrow{r_{\mathrm{PR}}}$ and $\overrightarrow{r_{\mathrm{EH}}}$. The range of the total diffusion $\left(r_{\mathrm{TD}}\right)$ in this scenario can be described by the vectorial addition formula $r_{\mathrm{TD}}^{2}=\left|\overrightarrow{r_{\mathrm{EH}}}\right|^{2}+\left|\overrightarrow{r_{\mathrm{PR}}}\right|^{2}+\left|\overrightarrow{r_{\mathrm{EH}}}\right|\left|\overrightarrow{r_{\mathrm{PR}}}\right| \cos \theta$, where $\theta$ is the angle between the two vectors. The total PR range in the $2 \mathrm{D}$ plane here is represented by $L_{\mathrm{PR}}$, which is related to the photon MFP in the film $\left(\lambda_{\text {film }}\right)$ by $L_{\mathrm{PR}}^{2}=2\langle M\rangle \lambda_{\text {film }}^{2} / 3$, i.e., in the $x$ - and $y$-directions, where $\lambda_{\text {film }}$ is given by $\lambda_{0}$ divided by the concentration ratio between the film and the standard solution (Supplementary Note 6). Averaging over all possible directions, the relation between the total diffusion length $\left(L_{\mathrm{D}}\right), \mathrm{EH}$ range $\left(L_{\mathrm{EH}}\right)$, and PR range $\left(L_{\mathrm{PR}}\right)$ is given by:

$$
L_{\mathrm{D}}^{2}=L_{\mathrm{EH}}^{2}+L_{\mathrm{PR}}^{2}
$$

Based on Eq. (6) and our diffusion length measurement and PR contribution results, we distinguished the quantitative contributions of $\mathrm{EH}$ and PR. The result is shown in Fig. 3b. Our results indicate that the EH process dominates diffusion mechanisms in PNC films, with a weaker, albeit considerable, contribution from the PR. Such long-range EH is unprecedented, given the isolated nature of the NCs separated by long insulating ligands.

\section{Discussion}

Interestingly, the octyl-based PNCs show the longest EH range, followed by the hexyl- and oleyl-based systems. To rationalize our findings, we performed grazing incidence small X-ray scattering (GISAXS) measurements to investigate the particle arrangement in the films. Our results reveal out-of-plane stacking in all our PNC films (Fig. 3c), with a characteristic distance of $\sim 65 \AA$. Assuming that the PNCs are arranged in a hexagonal close-packed (HCP) structure, this value corresponds to a center-to-center interparticle distance of $\sim 79 \AA$ between NCs, similar for all our PNC films. Such invariance is assigned to the oleate ligand present in all samples, which is vital for the stability of the PNCs. This bulky ligand becomes the limiting factor for tuning the interparticle distance within the films. Since our PNC films have similar interparticle distances, we could conclude that the differences in the $\mathrm{EH}$ ranges in our $\mathrm{PNC}$ films do not originate from their trivial differences in the interparticle distance but rather from their intricate intrinsic photophysical properties. 
To delve deeper into the physics of EH in our PNC films, we confirmed the role played by one of the most common mechanisms, i.e., FRET. Within Förster theory, the Förster radius $R_{0}$ (i.e., the distance at which the transfer efficiency is 50\%) can be calculated in $\AA$ as ${ }^{36,37}$ :

$$
R_{0}=9.78 \times 10^{3}\left[\kappa^{2} n^{-4} \eta J\right]^{1 / 6}
$$

where $\kappa^{2}=2 / 3$ is the dipole orientation factor for an isotropic sample; $n=1.5$ is the medium refractive index (i.e., that of the alkylamine ligands ${ }^{36}$ ); $\eta$ is the PLQY of our PNC films; and $J$ is the overlap integral between the PL of the donor and absorption of the acceptor (in $\mathrm{cm}^{3} \mathrm{M}^{-1}$ ), defined as:

$$
J=\int_{0}^{\infty} f_{\mathrm{D}}(\lambda) \epsilon_{\mathrm{A}}(\lambda) \lambda^{4} \mathrm{~d} \lambda
$$

Here, $f_{D}(\lambda)$ is the normalized PL spectrum of the donor (area $=1) ; \epsilon_{\mathrm{A}}(\lambda)$ is the extinction coefficient of the acceptor (in $\mathrm{M}^{-1} \mathrm{~cm}^{-1}$ ); and $\lambda$ is the wavelength (in $\mathrm{cm}$ ). The relation between $\sigma$ and $\epsilon_{\mathrm{A}}$ is presented in Supplementary Note 4 . The resulting $R_{0}$ values for our samples are summarized in Table 2.

Thus, our calculation shows remarkable values of $R_{0}$ in the PNCs, which are one order of magnitude larger than those in typical QD systems (tens of $\AA)^{36,37}$. This result implies an efficient FRET process that underpins the unprecedented robust EH in PNC films. To further confirm the role played by the FRET process in the observed $\mathrm{EH}$, we used these $R_{0}$ values estimated from independently measured parameters (Table 2) and tested their relationship with the extracted $L_{\mathrm{EH}}$ using the SmoluchowskiEinstein relation $^{38}$, which dictates the relation of the FRET-driven EH range $\left(L_{\mathrm{FRET}}\right)$ with $R_{0}$ :

$$
L_{\mathrm{FRET}}^{2}=A \frac{\tau_{f}}{\tau_{0}} \frac{R_{0}^{6}}{\tau^{4}} \propto \frac{R_{0}^{6}}{r^{4}}
$$

where $r$ is the inter-dipole distance; $A$ is a constant that accounts for the distribution of molecular separation; $\tau_{0}$ and $\tau_{\mathrm{f}}$ are the intrinsic and film exciton lifetimes, respectively. Figure $3 \mathrm{~d}$ shows a correlation between our

Table 2 FRET in our MAPbBr 3 PNCs. The PLQY in the film $\eta_{\text {film }}$, overlap integral $J$, and FRET range $R_{0}$ of the PNCs are given

\begin{tabular}{llll}
\hline & Hexyl & Octyl & Oleyl \\
\hline$\eta_{\text {film }}(\%)$ & $51 \pm 2$ & $57 \pm 2$ & $24 \pm 2$ \\
$J\left(10^{-10} \mathrm{~cm}^{3} \mathrm{M}^{-1}\right)$ & 1.04 & 0.95 & 1.34 \\
$R_{0}(\AA)$ & 135 & 136 & 124 \\
\hline
\end{tabular}

measured $L_{\mathrm{EH}}$ and $R_{0}$ fitted with the SmoluchowskiEinstein relation. Using our estimated $R_{0}$ values and assuming $79 \AA$ inter-particle distance, we obtained a proportionality constant of $\sim 500$ [underestimation of $R_{0}$ by a factor of $\sim 2.8$ times by eq. (7) assuming $A=1$ and $\tau_{0}=\tau_{\mathrm{f}}$, and FRET rates (i.e., $\tau_{\mathrm{FRET}} \propto R_{0}^{6}$ ) by a factor of $\sim 500]$. Such underestimation has also been reported in CdSe QD films, where the calculated $\tau_{\text {FRET }}$ underestimate the actual experimental results by $1-2$ orders of magnitude ${ }^{39}$. We believe that a more accurate and possibly quantitative model should not only account for the statistical distribution of the acceptors in the thin film, but also consider the presence of higher multipolar order contributions. However, this is beyond the scope of the current work. Furthermore, there could also be some minor contributions from other processes (e.g., exciton delocalization and Marcus-like charge transfer). However, the presence of a linear correlation between the calculated $R_{0}^{6}$ and $L_{\mathrm{EH}}^{2}$ confirms that FRET is driving the long-range $\mathrm{EH}$ process in PNC films.

In summary, we uncover unprecedented long exciton diffusion lengths exceeding $1 \mu \mathrm{m}$ in PNC films with magnitudes beyond their quantum sizes. Such long-range neutral exciton diffusion corresponds to mobilities up to $10 \pm 2 \mathrm{~cm}^{2} \mathrm{~V}^{-1 \mathrm{cs}} \mathrm{s}^{-1}$, surprisingly outpacing the charge carrier diffusion in the 3D counterparts. Through phenomenological modeling and use of colloidal suspensions as a playground to tune the concentration of the NCs, we distinguish the role of PR and inter-NC EH in PNC films. On a more fundamental level, our method provides a reliable and straightforward way to quantify the exciton transport in nanostructured systems. We discover that the long-range energy transport in PNC films is dominated by FRET. Considering the high PLQY of PNC systems, our findings demonstrate the enormous potential of LHP nanostructures not only for conventional optoelectronic applications (i.e., LEDs) but also for the emerging field of excitonic devices (e.g., exciton transistors ${ }^{40-42}$ ). Furthermore, the achievement of long-range energy transport is a step towards the implementation of biologically inspired solar cell $\operatorname{architectures}^{43}$, where a robust and long-range excitonic transport is key to enable high conversion efficiencies.

\section{Materials and Methods \\ Synthesis of PNCs}

Except for methylammonium bromide (MABr) purchased from Greatcell Solar Material, all reagents were purchased from Sigma-Aldrich. The organic and inorganic salt precursors were stored in a dry $\mathrm{N}_{2}$ glovebox, and all other reagents were stored in ambient conditions. The syntheses were carried out in ambient conditions under a fume hood.

$\mathrm{MAPbBr}_{3} \mathrm{PNCs}$ were synthesized by adapting the protocol previously reported by Veldhuis et al. (Ref. ${ }^{18}$ ). 
Briefly, a precursor stock solution was prepared by completely dissolving $117.4 \mathrm{mg}$ of lead bromide $\left(\mathrm{PbBr}_{2}\right)$ and $35.8 \mathrm{mg}$ of $\mathrm{MABr}$ in $2 \mathrm{~mL}$ of $\mathrm{N}, \mathrm{N}$-dimethylformamide (DMF, 99.8\% anhydrous). The different NCs were prepared by swift injection of this stock solution $(150 \mu \mathrm{L})$ into an antisolvent solution under vigorous and homogeneous stirring. For the Hexyl-MAPbBr ${ }_{3} \mathrm{PNCs}$, the antisolvent solution was prepared by adding $1 \mathrm{~mL}$ of oleic acid (OAc, $70 \%), 1 \mathrm{~mL}$ of benzyl alcohol (BzOH, 99.8\%), and $8 \mu \mathrm{L}$ of hexylamine to $5 \mathrm{~mL}$ of toluene. For the Octyl- $\mathrm{MAPbBr}_{3}$ PNCs, the antisolvent solution was prepared by adding $1 \mathrm{~mL}$ of oleic acid (OAc, 70\%), $1 \mathrm{~mL}$ of benzyl alcohol $(\mathrm{BzOH}, 99.8 \%)$, and $20 \mu \mathrm{L}$ of octylamine to $5 \mathrm{~mL}$ of toluene. For the Oleyl-MAPbBr 3 PNCs, the antisolvent solution was prepared by adding $1.5 \mathrm{~mL}$ of oleic acid (OAc, 70\%), $1 \mathrm{~mL}$ of benzyl alcohol (BzOH, 99.8\%), and $35 \mu \mathrm{L}$ of oleylamine to $5 \mathrm{~mL}$ of toluene.

After the injection, a green/yellow suspension was obtained. Purification of the crude material was performed using two centrifugation-redispersion steps at $12000 \mathrm{rpm}$ and $4000 \mathrm{rpm}$. Subsequently, the precipitated NCs were dispersed in $1.5 \mathrm{~mL}$ of anhydrous toluene and stored at $4{ }^{\circ} \mathrm{C}$.

\section{PL imaging measurement}

The PL imaging measurement was performed using a home-built microscope setup, as shown in Figure S6. The pump laser used was a $473 \mathrm{~nm}$ continuous wave laser. The microscope objective used was a 50X Mitutoyo Plan Apo Infinity Corrected Long WD Objective. The PL image was captured by using a PCo.edge 4.2 sCMOS camera. An illustration of the setup is shown in Figure S7. Size scaling of the microscope image was performed using a calibration slide. The pump image was collected on a clean glass slide with an additional neutral density filter to prevent saturation. The PL image was collected with an additional Semrock $488 \mathrm{~nm}$ long-pass filter. No leakage of pump scattering was detected when tested with a non-emitting sample. The pump laser power and camera integration time were kept constant throughout all measurements. The validity of the setup was confirmed with tests on the CdSe/ZnS core-shell QD system (see Supplementary Note 7).

\section{Linear absorption measurement}

The linear absorption measurement was performed with a commercial Shimadzu UV-3600i UV-VIS spectrometer.

\section{Steady-state and time-resolved PL measurement}

The steady-state PL and time-resolved PL measurements were performed using our home-built setup, powered by an $\sim 50$ fs Coherent LIBRA, with a repetition rate of $1 \mathrm{kHz}$. A $400 \mathrm{~nm}$ pump was generated from an $800 \mathrm{~nm}$ fundamental beam using a BBO crystal. Shortpass filters were added to block the residual fundamental beams after generation. The emission from the samples was collected with a lens pair and directed either to a monochromator (Princeton Instrument SP2300i) and streak camera (Optronis) system for time-resolved PL measurement or to a monochromator (Princeton Instrument SP2300i) and CCD camera (Pixis 400b) system for steady-state PL measurement.

\section{PLQY measurement}

The PLQY measurements were performed using a commercial Fluorolog 3 spectrofluorometer with an iHR320 emission monochromator integrated with a Quanta-Phi 6 integrating sphere.

\section{Acknowledgements}

We acknowledged Dr. Pio John S. Buenconsejo from the Facility for Analysis Characterization Testing and Simulation (FACTS), Nanyang Technological University, Singapore, for help with GISAXS measurements. This research/ project was supported by Nanyang Technological University under its start-up grants (M4080514, M4081630); the Ministry of Education under its AcRF Tier 1 grant (RG91/19) and Tier 2 grants (MOE2016-T2-1-034, MOE2017-T2-1-001, and MOE2017-T2-2-002); and the National Research Foundation (NRF) Singapore under its NRF Investigatorship (NRF-NRFI-2018-04) and Competitive Research Programme (NRF-CRP14-2014-03).

\section{Author contributions}

D.G. and M.R. conceived the idea. M.R. synthesized the samples. D.G. and M.R. performed the optical spectroscopy measurements and analysis of perovskite samples. Q.N. performed the AFM and GISAXS measurements and analysis. M.R. and J.W.M.L. performed the TEM measurement and analysis. D.G. and S.R. performed CdSe sample fabrication and measurement. T.C.S. led the project. All authors were involved in writing the manuscript.

\section{Data availability}

The data that support the findings of this study are openly available in DR-NTU (Data) at: https://doi.org/10.21979/N9/6QTW2E. Data are also available from the Corresponding Author upon reasonable request.

Conflict of interest

The authors declare that they have no conflict of interest.

Supplementary information is available for this paper at https:/doi.org/ 10.1038/s41377-020-00443-z.

Received: 19 July 2020 Revised: 13 November 2020 Accepted: 23 November 2020

Published online: 01 January 2021

\section{References}

1. Stranks, S. D. et al. Electron-hole diffusion lengths exceeding 1 micrometer in an organometal trihalide perovskite absorber. Science 342, 341-344 (2013).

2. Xing, G. C. et al. Long-range balanced electron- and hole-transport lengths in organic-inorganic $\mathrm{CH}_{3} \mathrm{NH}_{3} \mathrm{Pbl}_{3}$. Science 342, 344-347 (2013).

3. Sung, J. et al. Long-range ballistic propagation of carriers in methylammonium lead iodide perovskite thin films. Nat. Phys. 16, 171-176 (2020).

4. Guo, Z. et al. Long-range hot-carrier transport in hybrid perovskites visualized by ultrafast microscopy. Science 356, 59-62 (2017).

5. Deng, S. B. et al. Long-range exciton transport and slow annihilation in twodimensional hybrid perovskites. Nat. Commun. 11, 664 (2020).

6. Giovanni, D. et al. Ultrafast long-range spin-funneling in solutionprocessed Ruddlesden-Popper halide perovskites. Nat. Commun. 10, 3456 (2019).

7. Li, M. J. et al. Low threshold and efficient multiple exciton generation in halide perovskite nanocrystals. Nat. Commun. 9, 4197 (2018). 
8. de Weerd, C. et al. Efficient carrier multiplication in $\mathrm{CsPb}_{3}$ perovskite nanocrystals. Nat. Commun. 9, 4199 (2018).

9. Chen, J. S. et al. Cation-dependent hot carrier cooling in halide perovskite nanocrystals. J. Am. Chem. Soc. 141, 3532-3540 (2019).

10. Li, M. J. et al. Slow cooling and highly efficient extraction of hot carriers in colloidal perovskite nanocrystals. Nat. Commun. 8, 14350 (2017).

11. Kagan, C. R., Murray, C. B. \& Bawendi, M. G. Long-range resonance transfer of electronic excitations in close-packed CdSe quantum-dot solids. Phys. Rev. B 54, 8633-8643 (1996).

12. Kagan, C. R. et al. Electronic energy transfer in CdSe quantum dot solids. Phys. Rev. Lett. 76, 1517-1520 (1996).

13. Akselrod, G. M. et al. Subdiffusive exciton transport in quantum dot solids. Nano Lett. 14, 3556-3562 (2014).

14. Kang, J. \& Wang, L. W. High defect tolerance in lead Halide perovskite $\mathrm{CsPbBr}_{3}$. J. Phys. Chem. Lett. 8, 489-493 (2017).

15. Akkerman, Q. A. et al. Genesis, challenges and opportunities for colloidal lead halide perovskite nanocrystals. Nat. Mater. 17, 394-405 (2018).

16. Huang, $\mathrm{H}$. et al. Lead halide perovskite nanocrystals in the research spotlight: stability and defect tolerance. ACS Energy Lett. 2, 2071-2083 (2017).

17. Kim, Y. H. et al. Highly efficient light-emitting diodes of colloidal metal-halide perovskite nanocrystals beyond quantum size. ACS Nano 11, 6586-6593 (2017).

18. Veldhuis, S. A. et al. Benzyl alcohol-treated $\mathrm{CH}_{3} \mathrm{NH}_{3} \mathrm{PbBr}_{3}$ nanocrystals exhibiting high luminescence, stability, and ultralow amplified spontaneous emission thresholds. Nano Letters 17, 7424-7432 (2017).

19. Huang, $\mathrm{H}$. et al. Growth mechanism of strongly emitting $\mathrm{CH}_{3} \mathrm{NH}_{3} \mathrm{PbBr}_{3}$ perovskite nanocrystals with a tunable bandgap. Nat. Commun. 8, 996 (2017).

20. Tanaka, $\mathrm{K}$. et al. Comparative study on the excitons in lead-halide-based perovskite-type crystals $\mathrm{CH}_{3} \mathrm{NH}_{3} \mathrm{PbBr}_{3} \mathrm{CH}_{3} \mathrm{NH}_{3} \mathrm{Pbl}_{3}$. Solid State Commun. 127, 619-623 (2003).

21. Wang, Q. et al. Quantum confinement effect and exciton binding energy of layered perovskite nanoplatelets. AIP Adv. 8, 025108 (2018).

22. Zhang, Z. Y. et al. The role of trap-assisted recombination in luminescent properties of organometal halide $\mathrm{CH}_{3} \mathrm{NH}_{3} \mathrm{PbBr}_{3}$ perovskite films and quantum dots. Sci. Rep. 6, 27286 (2016).

23. Lee, E. M. Y. \& Tisdale, W. A. Determination of exciton diffusion length by transient photoluminescence quenching and its application to quantum dot films. J. Phys. Chem. C 119, 9005-9015 (2015).

24. Herz, L. M. Charge-carrier mobilities in metal halide perovskites: fundamental mechanisms and limits. ACS Energy Lett. 2, 1539-1548 (2017).

25. Pazos-Outón, L. M. et al. Photon recycling in lead iodide perovskite solar cells. Science 351, 1430-1433 (2016).
26. Brenes, R. et al. Benefit from photon recycling at the maximum-power point of state-of-the-art perovskite solar cells. Phys. Rev. Appl. 12, 014017 (2019).

27. Motti, S. G. et al. Heterogeneous photon recycling and charge diffusion enhance charge transport in quasi-2D lead-halide perovskite films. Nano Lett 19, 3953-3960 (2019).

28. Gan, Z. X. et al. The dominant energy transport pathway in halide perovskites: photon recycling or carrier diffusion? Adv. Energy Mater. 9, 1900185 (2019).

29. Wang, Y. P. et al. Photon transport in one-dimensional incommensurately epitaxial CsPbX 3 arrays. Nano Lett. 16, 7974-7981 (2016).

30. Dursun, I. et al. Efficient photon recycling and radiation trapping in cesium lead halide perovskite waveguides. ACS Energy Lett. 3, 1492-1498 (2018).

31. Bowman, A. R. et al. Quantifying photon recycling in solar cells and lightemitting diodes: absorption and emission are always key. Phys. Rev. Lett. 125, 067401 (2020).

32. Cho, $\mathrm{C}$. et al. The role of photon recycling in perovskite light-emitting diodes. Nat. Commun. 11, 611 (2020).

33. Turro, N. J. Energy transfer processes. in Photochemical Processes in Polymer Chemistry-2 (ed Smets, G.) (Pergamon, 1977), 405-429.

34. Andrews, D. L., Curutchet, C. \& Scholes, G. D. Resonance energy transfer: beyond the limits. Laser Photonics Rev. 5, 114-123 (2011).

35. Olaya-Castro, A. \& Scholes, G. D. Energy transfer from Förster-Dexter theory to quantum coherent light-harvesting. Int. Rev. Phys. Chem. 30, 49-77 (2011).

36. Righetto, M. et al. Engineering interactions in QDs-PCBM blends: a surface chemistry approach. Nanoscale 10, 11913-11922 (2018).

37. Stewart, M. H. et al. Competition between Förster resonance energy transfer and electron transfer in stoichiometrically assembled semiconductor quantum dot-fullerene conjugates. ACS Nano 7, 9489-9505 (2013).

38. Mikhnenko, O. V., Blom, P. W. M. \& Nguyen, T. Q. Exciton diffusion in organic semiconductors. Energy Environ. Sci. 8, 1867-1888 (2015).

39. Jolene Mork, A., Weidman, M. C., Prins, F. \& Tisdale, W. A. Magnitude of the Förster Radius in Colloidal Quantum Dot Solids. J. Phys. Chem. C 118, 13920-13928 (2014).

40. Unuchek, D. et al. Room-temperature electrical control of exciton flux in a van der Waals heterostructure. Nature 560, 340-344 (2018).

41. High, A. A. et al. Exciton optoelectronic transistor. Opt. Lett. 32, 2466-2468 (2007).

42. Kuznetsova, Y. Y. et al. All-optical excitonic transistor. Opt. Lett. 35, 1587-1589 (2010).

43. Brédas, J. L., Sargent, E. H. \& Scholes, G. D. Photovoltaic concepts inspired by coherence effects in photosynthetic systems. Nat. Mater. 16, 35-44 (2017). 